\title{
Ejercicio lúdico orientado a la apropiación de los Objetivos de Desarrollo Sostenible de la ONU
}

Design of a game focused on the appropriation of the UN Sustainable Development Goals

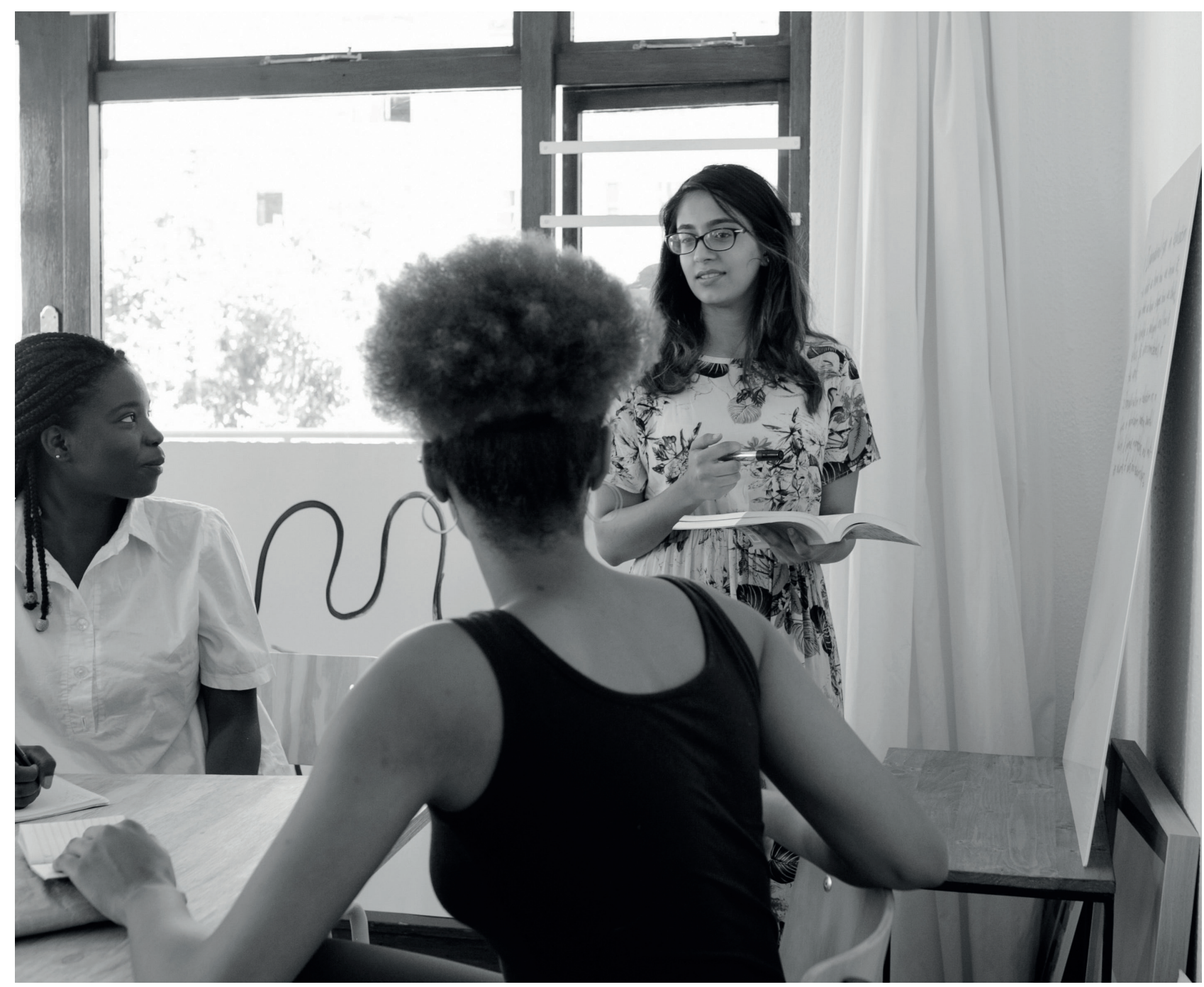




\title{
Ejercicio lúdico orientado a la apropiación de los Objetivos de Desarrollo Sostenible de la ONU ${ }^{1}$
}

\author{
Design of a game focused on the appropriation of the UN \\ Sustainable Development Goals
}

\author{
Mariana Acuña-Rangel², Mileidy Alvarez-Melgarejo³, Nicolás Hernández-Durán ${ }^{4}$
}

Artículo recibido en abril 27 de 2020; artículo aceptado en julio 03 de 2020

Este artículo puede compartirse bajo la Licencia Creative Commons Atribución-NoComercial-Compartirlgual 4.0 Internacional y se referencia usando el siguiente formato: Acuña-Rangel, M., Alvarez-Melgarejo, M. y Hernández-Durán, N. (2021). Ejercicio lúdico orientado a la apropiación de los Objetivos de Desarrollo Sostenible de la ONU. I+D Revista de Investigaciones, 16(1), 9-16. http://dx.doi. org/10.33304/revinv.v16n1-2021001.

\begin{abstract}
Resumen
El desarrollo sostenible es un tema de interés mundial, dado que busca proteger los recursos de las generaciones futuras. Es por esto que el Programa de las Naciones Unidas para el Desarrollo hace un llamado a la población a hacer uso de la creatividad y el conocimiento para proteger el planeta y garantizar que todas las personas gocen de paz y prosperidad para el año 2030, por medio del apoyo al cumplimiento de los Objetivos de Desarrollo Sostenible. Por tanto, en este artículo se describe el proceso mediante el cual se diseña una lúdica orientada a la promoción de los mencionados objetivos entre estudiantes de educación superior. La metodología usada fue el design thinking, con participación de integrantes del Laboratorio de Innovación Educativa GALEA. Los resultados permiten evidenciar que es factible diseñar una actividad que promueva la apropiación de los 17 Objetivos de Desarrollo Sostenible, y abre un espacio a futuro para la aplicación de la lúdica en entornos de clases, así como para su evaluación de impacto.
\end{abstract}

Palabras clave: Desarrollo sostenible, gamificación, lúdicas, ODS.

\begin{abstract}
Sustainable development is an issue of global interest, since it seeks to protect the resources of future generations, which is why the United Nations Development Program calls on the population to make use of creativity and knowledge to protect the planet and ensure that all people enjoy peace and prosperity by 2030, by supporting the achievement of the Sustainable Development Goals. The following article describes the process by which a game is designed to promote the SDGs among higher education students. The methodology used was design thinking, with

\footnotetext{
'Artículo de investigación, de enfoque cualitativo, resultado de un ejercicio de investigación y diseño como parte de la labor de enseñanza que se realiza en el Laboratorio GALEA, perteneciente al área de ciencias sociales, subárea de gestión organizacional y gerencia de proyectos, desarrollado en el Grupo de Investigación Finance \& Management, fue financiado por la Universidad Industrial de Santander (Bucaramanga, Colombia). Dirección: carrera 27 calle 9 Ciudad Universitaria, PBX: (57) (7) 6344000. Fecha de inicio: 30 de enero de 2020. Fecha de terminación: 17 de abril del 2020.

2 Estudiante de Ingeniería Industrial, Universidad Industrial de Santander. Grupo de Investigación Finance \& Management, Universidad Industrial de Santander (Bucaramanga, Colombia) Dirección: carrera 27 calle 9 Ciudad Universitaria, PBX: (57) (7) 6344000. https://orcid.org/0000-0001-98956809 Correo electrónico institucional: mariana.acuna@correo.uis.edu.co.

${ }^{3}$ Estudiante de Maestría en Administración, Universidad de Investigación y Desarrollo. Profesional adscrita al Grupo de investigación Finance \& Management, Universidad Industrial de Santander (Bucaramanga, Colombia) Dirección: carrera 27 calle 9 Ciudad Universitaria, PBX: (57) (7) 6344000. ORCID ID: https://orcid.org/0000-0003-1752-8023. Correo electrónico: mileidyalvarezm@gmail.com.

${ }^{4}$ Estudiante de Ingeniería Industrial, Universidad Industrial de Santander. Grupo de Investigación Finance \& Management, Universidad Industrial de Santander (Bucaramanga, Colombia) Dirección: carrera 27 calle 9 Ciudad Universitaria, PBX: (57) (7) 6344000. https://orcid.org/0000-0001-97549187. Correo electrónico institucional: nicolas.hernandez3@correo.uis.edu.co.
} 
Mariana Acuña-Rangel, Mileidy Alvarez-Melgarejo, Nicolás Hernández-Durán Ejercicio lúdico orientado a la apropiación de los Objetivos de Desarrollo Sostenible de la ONU

the participation of members of GALEA, an educational innovation laboratory. The results show that it is feasible to design an activity that promotes the appropriation of the 17 Sustainable Development Goals and opens a space in the future for the application of the game in classroom settings, as well as for its impact evaluation.

Keywords: Sustainable development, gamification, leisure, SDG.

\section{Introducción}

La sociedad actual se ve enfrentada diariamente a una serie de disyuntivas sobre la actuación frente a desafíos globales de índole económica, social y ambiental. Es por lo anterior que en septiembre del 2015, en el marco de las Naciones Unidas, los líderes mundiales adoptaron la Agenda 2030 para el Desarrollo Sostenible, que busca enfocar los esfuerzos de las comunidades hacia el cumplimiento de metas comunes que instalen prácticas sostenibles en el planeta (CEPAL, 2018). Para el logro de esta agenda se hace un llamado a las universidades en su rol de centros de educación, innovación, investigación y liderazgo (SDSN Australia/Pacific, 2017).

Uno de los mayores aportes de las universidades al cumplimiento de los Objetivos de Desarrollo Sostenible radica en el aprendizaje y la enseñanza, proporcionando a los estudiantes las habilidades y el conocimiento necesarios para proponer soluciones, además de impulsar las capacidades de los estudiantes en países en desarrollo (SDSN Australia/Pacific, 2017).

En el presente artículo se describe la creación de una lúdica enfocada en la enseñanza de los Objetivos de Desarrollo Sostenible para estudiantes universitarios, mediante el uso de la gamificación en el entorno educativo, para facilitar el desarrollo de habilidades blandas y la apropiación del conocimiento sobre la sostenibilidad.

\section{Objetivos para alcanzar el desarrollo sostenible}

Los Objetivos de Desarrollo Sostenible (ODS) fueron ideados como respuesta sistémica a problemáticas globales tan importantes como son la pobreza extrema, la hambruna, la desigualdad social y la degradación de medioambiente. Invitan, adicionalmente, a reforzar tanto la capacidad que tienen las instituciones para asumir estos desafíos como los esfuerzos para alcanzar las metas por medio de procesos de solidaridad global novedosos (Gómez, 2017).

Estos 17 ODS comprenden en total 169 metas, las cuales son de carácter integrado e indivisible, tienen un alcance mundial, y, además, su aplicación es universal. Estas características permiten que cada país o gobierno tenga la libertad de elegir la forma como incorpora los objetivos de la Agenda 2030 en sus políticas, planeación y estrategias nacionales (FAO, 2019).
Los objetivos buscan que las naciones alcancen "el desarrollo sostenible, el cual significa satisfacer las necesidades del presente sin comprometer la capacidad de las generaciones futuras de satisfacer las propias" (Brundtland, 1987). La anterior es la definición más citada de dicho concepto, y fue propuesta por la Comisión de las Naciones Unidas sobre Medio Ambiente y Desarrollo, conocida también como Comisión Bruntland, en 1987 (Gallopín, 2003).

Es importante enseñar a estudiantes universitarios sobre los ODS, ya que, por medio de ellos, se podrán lograr avances en cada una de las metas propuestas.

\section{Gamificación en el contexto educativo}

Para lograr lo anterior se deben utilizar estrategias de enseñanza disruptivas, entre las cuales se encuentra la gamificación, la cual utiliza elementos del juego en diferentes contextos, con el fin de aumentar la motivación y compromiso de los estudiantes hacia el aprendizaje (Acosta-Medina et al., 2020). A través de la gamificación se enriquece la experiencia educativa $y$, por ende, los estudiantes responderán positivamente al contenido (Deterding, 2012).

La gamificación aplica la mecánica y los elementos propios de un juego en procesos educativos, sacando provecho tanto de la predisposición psicológica de las personas que participan en juegos como de las propias bondades del juego para motivar y mejorar el comportamiento de los participantes (Kapp, 2012).

Entre los elementos que conforman la gamificación se encuentran la dinámica, la mecánica y los componentes. La mecánica hace referencia a los componentes básicos, las reglas y el funcionamiento del juego; la dinámica es la forma en que se pone en marcha la mecánica involucrando comportamientos y motivación, y, por último, los componentes, que son los recursos y herramientas con las cuales se diseña la actividad gamificada (Werbach y Hunter, 2012).

Estas nuevas técnicas de aprendizaje buscan desarrollar en los estudiantes actitudes y comportamientos de forma responsable (Aparicio et al., 2018), y así preparar futuros profesionales enfocados en la toma de decisiones fundamentadas, que les permita avanzar en el fortalecimiento y sostenibilidad de las empresas a las que pertenezcan en el futuro (Torres-Barreto et al., 2018). 
Mariana Acuña-Rangel, Mileidy Alvarez-Melgarejo, Nicolás Hernández-Durán

Ejercicio lúdico orientado a la apropiación de los Objetivos de Desarrollo Sostenible de la ONU

La gamificación se ha convertido en un tema con creciente interés para investigaciones científicas y en una herramienta con elevado potencial para ser aplicada en múltiples contextos educativos (Acosta-Medina et al., 2020; Escamilla et al., 2016).

\section{Formación integral universitaria}

Por otra parte, la educación universitaria implica no solo la adquisición de conocimientos y técnicas específicas para el ejercicio profesional, sino también la internalización de valores y actitudes que contribuyan a que el estudiante participe en la transformación de la sociedad (Ruiz, 2007).

El ambiente actual de globalización, complejidad y búsqueda de un futuro sostenible nos exige encaminarnos hacia el desarrollo humano integral, ya que de ello dependen muchas de las posibilidades de mejora de la calidad de vida de millones y millones de seres humanos (Tobón, 2010).

Para alcanzar una verdadera formación integral se debe tender al fortalecimiento de una personalidad ética, crítica y participativa, que interactúe con su entorno para que construya su identidad cultural (Marrero et al., 2018).

\section{Metodología}

Para la creación de la lúdica se usó de la metodología design thinking, utilizada para la generación de ideas innovadoras y que toma como centro la perspectiva de los usuarios finales. De esta forma se pueden detectar problemas y necesidades, así como ofrecer soluciones efectivas para cada una de ellas (Brown, 2008).

Este estudio es de tipo descriptivo y de enfoque no experimental, en cuanto se indican el proceso y los pasos seguidos para el diseño de la lúdica sin realizar experimento alguno. Se toman como referencia los pasos propuestos por IDEO (2014), pero se los adapta a la realidad particular de este proyecto de ideación; en este sentido, los cinco pasos propuestos originalmente fueron agrupados en tres.

\section{Selección del enfoque y población objetivo}

En este se agrupan los dos primeros pasos del design thinking, empatizar y definir, en los que se busca, respectivamente, comprender a los usuarios de la actividad y definir las características o necesidades de dichos individuos (Steinbeck, 2011).

La selección del enfoque de la lúdica se llevó a cabo por medio de la revisión del portafolio de programas de la Vicerrectoría de Investigación y Extensión de una universidad de Santander, identificando las temáticas y tendencias de investigaciones actuales, enfocadas en la generación de nuevo conocimiento y en la ejecución de acciones que aporten al desarrollo sostenible (Vicerrectoría de Investigación y Extensión, 2020). Dado que la población en estudio son los estudiantes de pregrado de la misma institución, al tratarse de los beneficiarios de la entidad que acoge el desarrollo de este estudio, se concluye que este es un tema de interés para ellos. Por lo anterior, se decide que el enfoque de la actividad lúdica gamificada sean los Objetivos de Desarrollo Sostenible, en un esfuerzo por generar conciencia social y dinamizar este tópico en la comunidad educativa.

\section{Proceso de ideación}

Con base en la fase de ideación propuesta por IDEO (2014), que pretende generar la mayor cantidad de ideas creativas posibles (Castillo-Vergara et al., 2014), en esta etapa el equipo de formulación, compuesto por estudiantes del programa de Ingeniería Industrial e integrantes del Laboratorio GALEA de innovación educativa, realizó una lluvia estructurada de ideas. Esta es una herramienta que potencia la creatividad en un grupo de personas, facilitando la intervención múltiple y voluntaria de los participantes (Herrán, 2011).

A través de esta técnica, se obtuvo una gran cantidad de ideas, las cuales fueron agrupadas en categorías generales que tuvieron como resultado el organizar la lúdica en retos cortos que se enfoquen en alguno de los 17 ODS, para que, al participar en alguno de estos, los estudiantes fomenten sus habilidades blandas mientras se contextualizan con cada objetivo. Adicionalmente, se propuso utilizar el material didáctico empleado para otras lúdicas del Laboratorio GALEA, con modificaciones menores, con el fin de reducir el consumo de nuevos recursos y apropiarse desde el inicio del enfoque sostenible de la actividad.

La ideación se llevó a cabo durante dos sesiones. La primera para definir la mecánica general, lo que produjo como resultado la división de la lúdica en cuatro momentos: explicación inicial, desarrollo de retos, presentación de propuestas y retroalimentación final. La segunda sesión tuvo como fin formular los retos y decidir en qué objetivos específicos se iban a enfocar para posteriormente estructurar las ideas recogidas durante las dos lluvias de ideas.

\section{Creación del prototipo}

En esta etapa se realiza el prototipo de la actividad, convirtiendo en físicas las ideas resultantes de la etapa anterior. En este punto, se realizó la búsqueda y selección de los materiales a utilizar en cada momento de la lúdica, los cuales se describen en el apartado "Materiales de la lúdica". El prototipo final fue presentado a los integrantes del Laboratorio GALEA, quienes hicieron una revisión de cada reto y propusieron sugerencias respecto al momento de la explicación inicial, con base 
Mariana Acuña-Rangel, Mileidy Alvarez-Melgarejo, Nicolás Hernández-Durán Ejercicio lúdico orientado a la apropiación de los Objetivos de Desarrollo Sostenible de la ONU

en su experiencia aplicando lúdicas para estudiantes universitarios. Es importante resaltar que el alcance de este artículo solo incluye la creación del ejercicio lúdico; por tanto, no se presenta la aplicación de la etapa final que consiste en la evaluación del ejercicio lúdico, la cual se espera llevar a cabo en sesiones en próximos meses para verificar el impacto de la lúdica en la apropiación de los ODS.

\section{Resultados}

Siguiendo la metodología de design thinking se logró el diseño de la lúdica, que se describe en los párrafos siguientes.

En la etapa de explicación inicial se expondrá a los estudiantes la mecánica de la actividad, iniciando con una breve introducción sobre los Objetivos de Desarrollo Sostenible y la importancia de tomar acciones sobre estos. El objetivo de la actividad es que se diseñe un producto o servicio que trabaje en pro del cumplimiento de uno o más ODS. Para el desarrollo de retos se dividirá a los participantes en tres grupos, lo que invita a los participantes a trabajar en equipo frente a una meta común: la construcción del producto o servicio. Se desarrollaron cinco retos, los cuales serán enfrentados por uno, dos o tres miembros de cada grupo, simultáneamente, según como lo requiera cada uno de ellos.

- Reto 1. En las profundidades: Este reto se enfoca en el ODS 14: Vida submarina. En este deben participar dos miembros de cada grupo, uno como pescador y otro como guía. El participante que se desempeñe como guía deberá dar instrucciones verbales a su compañero de equipo, para que este consiga pescar la mayor cantidad de basura de un ecosistema marino. Se otorgarán puntos positivos por cada basura extraída con satisfacción, y puntos negativos por pescar los animales pertenecientes al ecosistema. El grupo que tenga mayor número de puntos gana el reto.

- Reto 2. Supera los límites: Este reto se enfoca en el ODS 4: Educación de calidad. Para este se requiere que participen tres miembros de cada grupo. Cada uno se verá enfrentado a una limitación para el desarrollo de este reto, cuyo proceso es el siguiente: uno de los integrantes tendrá los ojos vendados e iniciará la actividad a ciertos metros de distancia de la mesa central del juego, a la cual se deberá acercar siguiendo órdenes de su segundo compañero, que tendrá un lápiz entre los labios que limitará su capacidad de habla. Cuando el primer integrante alcance a su compañero, deberá tomar un papel que contiene una operación matemática simbolizada por frutas, que deberá ser resuelta por el tercer integrante, que tendrá las manos atadas. Cuando este llegue a la respuesta correcta, su compañero, con el lápiz en los labios, debe guiar a aquel con los ojos vendados para que el último tome una ficha con el número de la solución al problema matemático, la cual en su reverso tiene una frase escrita. Luego de que el compañero con los ojos vendados tome la ficha correcta, se la debe entregar a aquel con el lápiz en los labios, para que le dicte la frase a su compañero con las manos atadas mientras este último escribe la frase. El grupo que termine primero gana el reto.

- Reto 3. En peligro de extinción: Este reto se enfoca en el ODS 15: Vida de ecosistemas terrestres. Para este reto solo se requiere la participación de un integrante de cada grupo, que será dotado de una serie de aros que deberá lanzar hacia un tablero con imágenes de diferentes animales, algunos de los cuales están en peligro de extinción. Cuando consiga "atrapar" a algún animal, deberá iniciar con la narración de una historia que incluya a dicha criatura. Si alguien ya previamente había atrapado a un animal e iniciado una narración, el próximo en acertar debe continuar la historia anterior e incluir como personaje al animal que atrapó, cuidando mantener el hilo conductor de la historia. Los animales en peligro de extinción brindan puntos positivos $y$, adicionalmente, se otorgan puntos por la creatividad de la historia narrada. Gana aquel equipo con más puntos.

- Reto 4: Agua pasó por aquí: Este reto se enfoca en el ODS 6: Agua limpia y saneamiento. En este deben participar dos integrantes de cada grupo, quienes deberán seleccionar un tanque que tiene una pregunta con tres opciones de respuesta para ser contestada correctamente. De lo contrario, cuando se llene el tanque de agua, esta saldrá sucia. Ganan los equipos que seleccionen la respuesta correcta.

- Reto 5: Sincroniza: Este reto se enfoca en el ODS 11: Ciudades y comunidades sostenibles. Acá participan dos integrantes de cada grupo. A cada uno se le entregará una hoja y un lápiz, y deberán ubicarse en lugares opuestos dentro del aula donde se desarrolle la lúdica. Se le indicará a cada uno que escriba cinco palabras que relacione con la categoría "ciudad sostenible". Al finalizar se leerán las palabras que escribió cada miembro y tendrán un punto por cada palabra que tengan en común ambos participantes. Gana el grupo con más puntos.

Cuando un equipo pierda un reto deberá girar una ruleta que incluye una serie de restricciones, la cual deberán aplicar al producto o servicio que estén diseñando dentro del grupo. Al finalizar los retos se les proporcionarán materiales para que construyan un prototipo de su propuesta.

En la etapa de presentación de propuestas, uno o más integrantes del grupo deberán exponer en dos minutos su producto o servicio y mencionar cómo este aporta al cumplimiento de los Objetivos de Desarrollo Sostenible. 
Mariana Acuña-Rangel, Mileidy Alvarez-Melgarejo, Nicolás Hernández-Durán

Ejercicio lúdico orientado a la apropiación de los Objetivos de Desarrollo Sostenible de la ONU

La propuesta mejor desarrollada será la ganadora de la actividad.

Para la retroalimentación, el encargado de dirigir la actividad describirá las actitudes positivas que notó en el desarrollo de la lúdica y resaltará cómo se reforzó en cada uno de ellos sus habilidades blandas, además de contextualizarlos sobre el desarrollo sostenible y sus 17 objetivos.

\section{Materiales de la lúdica}

Para cada uno de los retos se utiliza el material didáctico que se muestra en las Figuras 1, 2, 3 y 4, y que se describe a continuación:

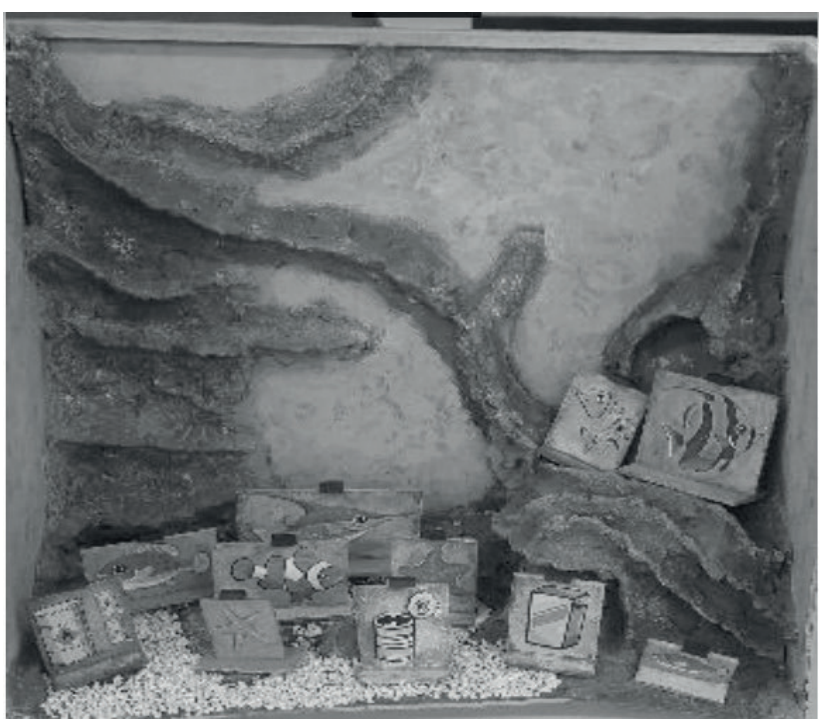

Figura 1. Ecosistema submarino usado en el Reto 1. Fuente: Laboratorio GALEA.

Adicionalmente se requiere para este reto lana, imanes y palos de pincho.

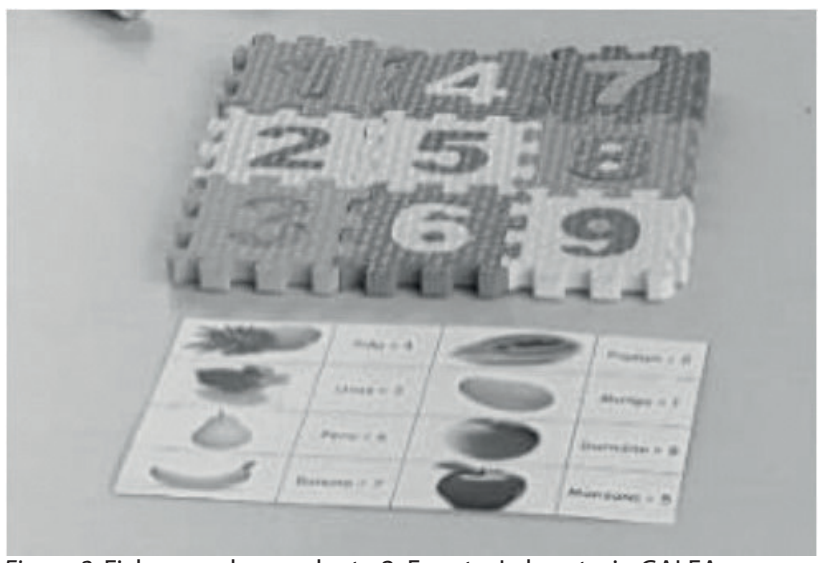

Figura 2. Fichas usadas en el reto 2. Fuente: Laboratorio GALEA

Para el reto dos se requieren, además, lápices, vendas y lana.

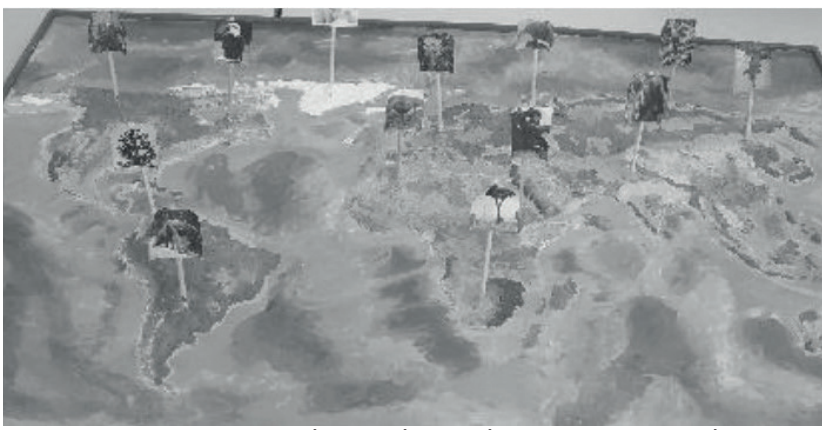

Figura 3. Mapa con animales usado en el reto 3. Fuente: Laboratorio GALEA.

En adición al mapa anterior, se requieren anillos de plástico que serán lanzados por los participantes para atrapar los animales.

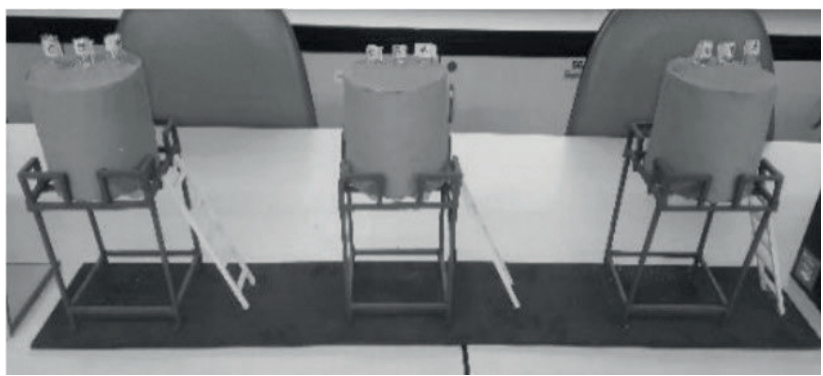

Figura 4. Tanques usados en el reto 4. Fuente: Laboratorio GALEA.

Para el desarrollo del prototipo se entregarán legos, plastilina, marcadores, cinta, tijeras, papel y palos de paleta.

\section{Conclusiones}

Siguiendo lo planteado por Burke (2014), este artículo plantea que la gamificación cumple un rol importante cuando se desea impulsar el cambio en comunidades, además de permitir el diseño de actividades disruptivas que traigan al salón de clase temas de importancia actual, para crear conciencia social y motivar la participación activa en proyectos para el bien común.

A través de la metodología planteada, se logró completar la ideación y prototipado de una actividad lúdica que tiene como objetivo difundir los Objetivos de Desarrollo Sostenible propuestos por la ONU entre los estudiantes universitarios, además de fortalecer las habilidades blandas en ellos, tales como trabajo en equipo, comunicación asertiva y liderazgo, entre otras.

El proceso de diseño de esta actividad lúdica permitió a los autores conocer un poco más sobre la importancia que tienen los objetivos de desarrollo sostenible para el país y para el mundo, así como el rol tan relevante de "desarrollar nuevas metodologías de enseñanza en las instituciones de educación que faciliten la enseñanza y el aprendizaje" (Lobo-Rueda et al., 2020). Además, el design thinking permitió obtener ideas creativas para llevar a 
Mariana Acuña-Rangel, Mileidy Alvarez-Melgarejo, Nicolás Hernández-Durán Ejercicio lúdico orientado a la apropiación de los Objetivos de Desarrollo Sostenible de la ONU

cabo el diseño de la actividad lúdica, sin perder de vista el objetivo de esta y la población con la cual será aplicada.

El paso a seguir es realizar la puesta en marcha de la actividad, aplicándola en la población objetivo; es decir, estudiantes de pregrado. Con la aplicación de la lúdica se espera promover la difusión y conocimiento sobre los ODS, así como corroborar el impacto que tienen las actividades lúdicas como esta en los procesos de enseñanza y aprendizaje de la comunidad estudiantil.

De cara al futuro, el Laboratorio de Innovación Educativa GALEA tiene un llamado no solo a seguir apoyando la creación de ambientes educativos diferentes a los tradicionales, facilitar la creatividad e incentivar a la comunidad educativa para que se involucre en proyectos de diseño y puesta en marcha de actividades lúdicas de impacto en los estudiantes, sino también a realizar evaluaciones de ejecución e impacto de estas actividades lúdicas, tales que permitan medir los efectos de los ejercicios de este tipo en la comunidad académica. $Y$ esto incluye no solo a estudiantes, sino a profesores y directivos, quienes juegan conjuntamente un papel en el desarrollo de una comunidad de aprendizaje renovada.

\section{Agradecimientos}

Agradecemos a la Universidad Industrial de Santander, por facilitarnos los escenarios para la realización de esta investigación. De igual forma, a la profesora Martha Liliana Torres Barreto, directora del Grupo de Investigación Finance \& Management y del Laboratorio GALEA de la Universidad Industrial de Santander, por su apoyo en el diseño de la lúdica, su supervisión y comentarios en la construcción de este manuscrito.

\section{Referencias}

Acosta-Medina, J. K., Torres-Barreto, M. L., AlvarezMelgarejo, M. y Paba-Medina, M. C. (2020). Gamificación en el ámbito educativo: Un análisis bibliométrico. I+D Revista de Investigaciones, 15(1), 30-39.

Aparicio, D., Torres-Barreto, M. y Alvarez-Melgarejo, M. (2018). Competencias ciudadanas desde un enfoque de gamificación. https://hal.archives-ouvertes.fr/ hal-01952203

Brown, T. (2008). Design Thinking. Harvard Business Review, 86(6), 84.

Brundtland, G. H. (1987). Informe de la Comisión Mundial sobre el Medio Ambiente y el Desarrollo: Nuestro futuro común. Documentos de Las Naciones, Recolección de Un..., 416.

Burke, B. (2014). Gamify: How Gamification Motivates People to Do Extraordinary Things. Gartner Inc.
Castillo-Vergara, M., Alvarez-Marin, A. y Cabana-Villca, R. (2014). Design Thinking: How to Guide Students and Business Entrepreneurs in the Application. Ingeniería Industrial, 35(3), 301-311.

CEPAL. (2018). La Agenda 2030 y los Objetivos de Desarrollo Sostenible: Una oportunidad para América Latina y el Caribe. Naciones Unidas https://repositorio.cepal. org/handle/11362/40155.4

Herrán, A. (2011). Técnicas didácticas para una enseñanza más formativa. En N. Álvarez Aguilar y R. Cardoso Pérez (Coords.), Estrategias y metodologías para la formación del estudiante en la actualidad. Universidad de Camagüey. http://radicaleinclusiva. com/wp-content/uploads/2018/01/teuniv.pdf

Deterding, S. (2012). Gamification: Designing for Motivation. Iteractions, 19(4), $14 . \mathrm{https} / / /$ doi. org/10.1145/2212877.2212883

Escamilla, J., Fuerte, K., Venegas, E., Fernández, K., Elizondo, J. y Román, R. (2016). Gamificación. En Observatorio de Innovación Educativa del Tecnológico de Monterrey (pp. 1-36). http://eduteka.icesi.edu.co/ pdfdir/edutrends-gamificacion.pdf

FAO. (2019). El apoyo de la FAO para alcanzar los Objetivos de Desarrollo Sostenible en América del Sur. Panorama actual. Santiago de Chile, 1-76. http:// www.fao.org/3/ca3884es/ca3884es.pdf

Gallopín, G. (2003). Sostenibilidad y desarrollo sostenible: un enfoque sistémico. Cepal.

Gómez, C. (2017). Objetivos de Desarrollo Sostenible (ODS): una revisión crítica. Papeles de relaciones ecosociales y cambio global, (140), 107-118.

IDEO. (2014). Design Thinking en Español. Dinngo http:// www.designthinking.es/inicio/

Kapp, K. M. (2012). The Gamification of Learning and Instruction: Game-Based Methods and Strategies for Training and Education. John Wiley \& Sons. https:// doi.org/10.1145/2207270.2211316

Lobo-Rueda, M. A., Paba-Medina, M. C. y Torres-Barreto, M. L. (2020). Análisis descriptivo de experiencias gamificadas para enseñanza y aprendizaje en educación superior en ingeniería. Revista ESPACIOS, 41(16), 21. https://www.revistaespacios.com/ a20v41n16/20411621.html

Marrero, O., Mohamed, R. y Xifra, J. (2018). Habilidades blandas: necesarias para la formación integral del estudiante universitario. Revista Científica Ecociencia, 5, 1-18. 
Mariana Acuña-Rangel, Mileidy Alvarez-Melgarejo, Nicolás Hernández-Durán

Ejercicio lúdico orientado a la apropiación de los Objetivos de Desarrollo Sostenible de la ONU

Ruiz, L. (2007). Formación integral: Desarrollo intelectual, emocional, social y ético de los estudiantes. Revista Universidad de Sonora, (1), 11-13.

SDSN Australia/Pacific. (2017). Cómo empezar con los ODS en las universidades: una guía para las universidades, los centros de educación superior y el sector académico. Sustainable Development Solutions Network - Australia/Pacific. https://redssdsn.es/wp-content/uploads/2017/02/Guia-ODSUniversidades-1800301-WEB.pdf

Steinbeck, R. (2011). El «design thinking» como estrategia de creatividad en la distancia. Comunicar, 19(37), 2735. https://doi.org/10.3916/C37-2011-02-02

Tobón, S. (2010). Formación integral y competencias. Pensamiento complejo, currículo, didáctica y evaluación. (3. ${ }^{a}$ ed.). Centro de Investigación en Formación y Evaluación CIFE. Ecoe Ediciones, 2010.

Torres-Barreto, M. L., Alvarez-Melgarejo, M. y Ardila, T. Y. (2018). Laboratorio GALEA: un espacio en transformación e innovación para la enseñanzaaprendizaje. https://hal.archives-ouvertes.fr/hal01894004

Vicerrectoría de Investigación y Extensión. (2020). Portafolio de Programas 2020. https://n9.cl/tn1u

Werbach, K. y Hunter, D. (2012). For the Win: How Game Thinking Can Revolutionize Your Business. Wharton Digital Press. 